\title{
Non-vanishing for Koszul cohomology of curves
}

\author{
M. Aprodu and J. Nagel
}

Abstract. We study the relationship between rank $p+2$ Koszul classes and rank 2 vector bundles on a smooth curve. We show that every rank $p+2$ Koszul class is obtained from a rank 2 vector bundle and give an explicit nonvanishing theorem for Koszul classes arising in this way.

Mathematics Subject Classification (2000). 13D02, $14 \mathrm{H} 60$.

Keywords. Syzygies, Koszul cohomology, complex curves.

\section{Introduction}

Let $X$ be a smooth complex projective variety. The geometry of $X$ is reflected in the behaviour of the Koszul cohomology groups $K_{p, q}(X, L)$ introduced by Green [4], more specifically the vanishing/nonvanishing of certain Koszul cohomology groups. The fundamental result in this direction is the nonvanishing theorem of Green-Lazarsfeld [5]. This theorem states that if a line bundle $L$ admits a decomposition $L=L_{1} \otimes L_{2}$ with $r_{i}=h^{0}\left(X, L_{i}\right)-1 \geq 1(i=1,2)$ then $K_{r_{1}+r_{2}-1,1}(X, L) \neq 0$. Voisin [9, (1.1)] has given a different proof of this result under the hypothesis that $L_{1}$ and $L_{2}$ are globally generated.

The aim of this note is to give a more geometric approach to this type of problems. The starting point is the following construction due to Voisin. Given a rank two vector bundle $E$ on $X$ with determinant $L$, Voisin [11, (2.22)] defined a homomorphism

$$
\varphi: S^{p} H^{0}(X, E) \otimes \bigwedge^{p+2} H^{0}(X, E) \rightarrow \bigwedge^{p} H^{0}(X, L) \otimes H^{0}(X, L) .
$$

By [11, Lemma 5], this homomorphism produces elements of $K_{p, 1}(X, L)$. If we take $E=L_{1} \oplus L_{2}$, we get back the classes constructed by Green and Lazarsfeld. As one of the referees pointed out to us, Koh and Stillman [7] had generalised the Green-Lazarsfeld construction before from a different point of view.

Recall that the rank of a Koszul class $\gamma \in K_{p, 1}(X, L)$ is the minimal dimension of a linear subspace $W \subset H^{0}(X, L)$ such that $\gamma$ is represented by an element in $\bigwedge^{p} W \otimes H^{0}(X, L)$; cf. [6, Definition 2.2]. (Note that the subspace $W$ is uniquely 
determined if $p \geq 2$.) By definition, the Koszul classes constructed in this paper are of rank $p+2$ if the vector bundle $E$ is indecomposable.

Section 3 contains the main results of this paper. We first give a necessary and sufficient condition for nonvanishing of Koszul classes on smooth curves obtained from rank 2 vector bundles (Theorem 3.1). This result generalises the nonvanishing theorem of Green-Lazarsfeld in the case of curves. Our second main result, Theorem 3.4, states that every rank $p+2$ Koszul class on a smooth curve comes from a rank two vector bundle. This theorem is a generalisation of [6, Theorem 6.7].

\section{Preliminaries}

2.1. The method of Voisin. Let $E$ be a rank two vector bundle on a smooth projective variety $X$ defined over an algebraically closed field $k$ of characteristic zero. Write $L=\operatorname{det} E$ and $V=H^{0}(X, L)$, and let

$$
d: \bigwedge^{2} H^{0}(X, E) \rightarrow V
$$

be the determinant map. Given $t \in H^{0}(X, E)$, define a linear map

$$
d_{t}: H^{0}(X, E) \rightarrow V
$$

by $d_{t}(u)=d(t \wedge u)$, and choose a subspace $U \subset H^{0}(X, E)$ with $U \cap \operatorname{ker}\left(d_{t}\right)=0$. Suppose that $\operatorname{dim}(U)=p+2$ with $p \geq 1$, and put $W=d_{t}(U) \cong U$. The restriction of $d$ to $\bigwedge^{2} U$ defines a map $\bigwedge^{2} U \rightarrow V$, which we can view as an element of

$$
\bigwedge^{2} U^{\vee} \otimes V \cong \bigwedge^{p} U \otimes V
$$

Let

$$
\gamma \in \bigwedge^{p} W \otimes V \subset \bigwedge^{p} V \otimes V
$$

be the image of this element under the map $d_{t}$.

Following Voisin $[11,(2.22)]$, we prove that $\gamma$ defines a Koszul class in $K_{p, 1}(X, L)$. To this end, we make the previous construction explicit using coordinates. If we choose a basis $\left\{e_{1}, \ldots, e_{p+3}\right\}$ of $\langle t\rangle \oplus U \subset H^{0}(X, E)$ such that $e_{1}=t$, we have

$$
\begin{aligned}
\left.\gamma=\sum_{i<j}(-1)^{i+j} d\left(t \wedge e_{2}\right) \wedge \cdots \wedge d \widehat{\left(t \wedge e_{i}\right.}\right) \wedge \cdots \\
\left.\quad \cdots \wedge d \widehat{\left(t \wedge e_{j}\right.}\right) \wedge \cdots \wedge d\left(t \wedge e_{p+3}\right) \otimes d\left(e_{i} \wedge e_{j}\right) .
\end{aligned}
$$

As in [11] one shows that the image of the $\gamma$ by the Koszul differential

$$
\delta: \bigwedge^{p} V \otimes H^{0}(X, L) \rightarrow \bigwedge^{p-1} V \otimes S^{2} H^{0}(X, L)
$$


equals

$$
\begin{aligned}
& \left.\sum_{i<j<k}(-1)^{i+j+k} d\left(t \wedge e_{2}\right) \wedge \cdots \wedge d \widehat{\left(t \wedge e_{i}\right.}\right) \wedge \cdots \\
& \quad \cdots \wedge d\left(\widehat{\left(t \wedge e_{j}\right.}\right) \wedge \cdots \wedge d \widehat{\left(t \wedge e_{k}\right)} \wedge \cdots \wedge d\left(t \wedge e_{p+3}\right) \\
& \quad \otimes\left\{d\left(t \wedge e_{i}\right) d\left(e_{j} \wedge e_{k}\right)-d\left(t \wedge e_{j}\right) d\left(e_{i} \wedge e_{k}\right)+d\left(t \wedge e_{k}\right) d\left(e_{i} \wedge e_{j}\right)\right\}
\end{aligned}
$$

Lemma 2.1 (Voisin). Given four elements $w_{1}, w_{2}, w_{3}, w \in H^{0}(X, E)$ we have the relation

$$
d\left(w \wedge w_{1}\right) d\left(w_{2} \wedge w_{3}\right)-d\left(w \wedge w_{2}\right) d\left(w_{1} \wedge w_{3}\right)+d\left(w \wedge w_{3}\right) d\left(w_{1} \wedge w_{2}\right)=0
$$

in $H^{0}\left(X, L^{2}\right)$.

Proof. See [11, Lemma 5].

The previous lemma shows that $\gamma$ belongs to the kernel of the Koszul differential

$$
\delta_{X}: \bigwedge^{p} V \otimes H^{0}(X, L) \rightarrow \bigwedge^{p-1} V \otimes H^{0}\left(X, L^{2}\right) .
$$

Hence $\gamma$ defines a Koszul class $[\gamma]=\gamma(U, t) \in K_{p, 1}(X, L, W) \subseteq K_{p, 1}(X, L)$.

Remark 2.2. If $U^{\prime} \subset\langle t\rangle \oplus U \subset d_{t}^{-1}(W)$ is another lifting of $W$, then $\gamma(U, t)=$ $\gamma\left(U^{\prime}, t\right)$. In particular, if $\operatorname{ker}\left(d_{t}\right)=\mathbb{C}$. $t$ the given class only depends on $t$ and $W$; we write $[\gamma]=\gamma(W, t)$ in this case.

2.2. The method of Green-Lazarsfeld. Let $L_{1}, L_{2}$ be two line bundles on a smooth projective variety $X$ such that $r_{i}=h^{0}\left(X, L_{i}\right)-1 \geq 1(i=1,2)$. Write $L_{i}=$ $M_{i}+F_{i}$ with $M_{i}$ the mobile part and $F_{i}$ the fixed part. Let $B$ be the divisorial part of $F_{1} \cap F_{2}$. It is possible to choose $s_{i} \in H^{0}\left(X, L_{i}\right)$ such that $V\left(s_{1}, s_{2}\right)=B \cup Z$ with $\operatorname{codim}(Z) \geq 2$. Set $L=L_{1} \otimes L_{2}$, and put $t=\left(s_{1}, s_{2}\right) \in H^{0}\left(X, L_{1} \oplus L_{2}\right), W=$ $\operatorname{im}\left(d_{t}\right) \subset H^{0}(X, L(-B))$. By construction $h^{0}\left(X, \mathcal{O}_{X}(B)\right)=1$, hence $\operatorname{ker}\left(d_{t}\right)=\mathbb{C} . t$ and $\operatorname{dim} W=r_{1}+r_{2}+1$. By the previous discussion, we obtain a Koszul class $\gamma(W, t) \in K_{r_{1}+r_{2}-1,1}(X, L)$. We call such classes Green-Lazarsfeld classes.

Note that the rank of a Green-Lazarsfeld class is either $p+1$ or $p+2$. Classes of rank $p+1$ are of scrollar type; see e.g. [8] or [6, Corollary 5.2].

Definition 2.3. Given a nonnegative integer $k \geq 0$, let $K_{k, 1}(X, L)_{\mathrm{GL}} \subseteq K_{k, 1}(X, L)$ be the subspace generated by Green-Lazarsfeld classes for all decompositions $L=$ $L_{1} \otimes L_{2}$ with $k=r_{1}+r_{2}-1,\left(r_{1} \geq 1, r_{2} \geq 1\right)$. 
2.3. The method of Koh-Stillman. Voisin's method produces syzygies of rank $\leq p+2$. As we have seen in the previous subsection, rank $p+1$ syzygies are Green-Lazarsfeld syzygies of scrollar type. Rank $p+2$ syzygies can be obtained in the following way. Suppose that $L$ is a globally generated line bundle on a projective variety $X$, and let $[\gamma] \in K_{p, 1}(X, L)$ be a nonzero class represented by an element $\gamma \in \bigwedge^{p} W \otimes V$ with $\operatorname{dim} W=p+2$. We view $\gamma$ as an element in $\bigwedge^{2} W^{\vee} \otimes V \cong$ Hom $\left(\bigwedge^{2} W, V\right)$. Following [6, Proof of Theorem 6.1] we consider the map

$$
\gamma^{\prime}: \bigwedge^{2}(\mathbb{C} \oplus W)=W \oplus \bigwedge^{2} W \rightarrow V
$$

defined by taking the direct sum of $\gamma$ and the inclusion $W \hookrightarrow V$. If we choose a generator $e_{1}$ for the first summand and a basis $\left\{e_{2}, \ldots, e_{p+3}\right\}$ for $W$, we obtain a skew-symmetric $(p+3) \times(p+3)$ matrix $A$ by setting

$$
a_{i j}=\gamma^{\prime}\left(e_{i} \wedge e_{j}\right) .
$$

By construction, the inclusion $W \rightarrow V$ corresponds to the map $\gamma^{\prime}\left(e_{1} \wedge-\right)$. This allows us to identify $a_{1 j}$ and $e_{j}, 2 \leq j \leq p+3$. Let $\alpha$ be the image of $\gamma$ under the Koszul differential

$$
\delta: \bigwedge^{p} V \otimes V \rightarrow \bigwedge^{p-1} V \otimes S^{2} V .
$$

Writing this out, we obtain

$\alpha=\sum_{i<j<k}(-1)^{i+j+k} a_{12} \wedge \cdots \wedge \widehat{a_{1, i}} \wedge \cdots \wedge \widehat{a_{1, j}} \wedge \cdots \wedge \widehat{a_{1, k}} \wedge \cdots \wedge a_{1, p+3} \otimes \operatorname{Pf}_{1 i j k}(A)$.

As the elements $\left\{a_{12}, \ldots, a_{1, p+3}\right\}=\left\{e_{2}, \ldots, e_{p+3}\right\}$ are linearly independent, this expression is nonzero if and only if at least one of the Pfaffians $\operatorname{Pf}_{1 i j k}(A)$ is nonzero. Furthermore, since $\alpha$ maps to zero in $\bigwedge^{p-1} V \otimes H^{0}\left(X, L^{2}\right)$ the Pfaffians $\operatorname{Pf}_{1 i j k}(A)$ have to vanish on the image of $X$.

The preceding discussion shows that every rank $p+2$ syzygy arises from a skewsymmetric $(p+3) \times(p+3)$ matrix $A$ such that

(i) the elements $\left\{a_{12}, \ldots, a_{1, p+3}\right\}$ are linearly independent;

(ii) there exists a nonzero Pfaffian $\operatorname{Pf}_{1 i j k}(A)$;

(iii) the Pfaffians $\operatorname{Pf}_{1 i j k}(A)$ vanish on the image of $X$ in $\mathbb{P}\left(V^{\vee}\right)$.

This is exactly the method used by Koh and Stillman to produce syzygies; see [7, Lemma 1.3]

Remark 2.4. In the geometric setting of Section 2.1, let $Y$ be the image of $X$ in $\mathbb{P}\left(V^{\vee}\right)$. The expression (2) shows that the canonical isomorphism

$$
K_{p, 1}(X, L) \cong K_{p-1,2}\left(\mathbb{P}^{r}, \tau_{Y}, \mathcal{O}_{\mathbb{P}}(1)\right)
$$


maps the class $[\gamma]$ to the element $\alpha$ defined in (3). Moreover, if $d$ does not vanish on decomposable elements then $[\gamma] \neq 0$. Indeed, this condition is satisfied if and only if the matrix $A$ has no generalised zero; cf. [7, Definition (1.1)]. One then applies [loc. cit., Remark p. 122].

\section{Main results}

Theorem 3.1. Let $X$ be a smooth curve, let $L$ be a base-point free line bundle on $X$ and let $W \subset H^{0}(X, L)$ be a linear subspace. Put $B=\mathrm{Bs}(W)$, and let $t$ be a section of $H^{0}\left(X, \mathcal{O}_{X}(B)\right)$ vanishing on $B$. Consider an extension

$$
0 \rightarrow \mathcal{O}_{X}(B) \rightarrow E \rightarrow L(-B) \rightarrow 0
$$

such that

$$
W \subset\left(\operatorname{ker} H^{0}(X, L(-B)) \stackrel{\delta}{\longrightarrow} H^{1}\left(X, \mathcal{O}_{X}(B)\right)\right) .
$$

Then the Koszul classes $\gamma(U, t)$ defined in Section 2.1 are nonzero for all liftings $U$ of $W$ if and only if the extension (4) is non-split.

Proof. The proof proceeds in several steps. We use the notation of Section 2.1.

"Only if". Suppose that the extension (4) splits, hence $W \subset H^{0}(X, E)$ canonically. We then put $U=W$. In this case, one readily verifies that $d$ vanishes identically on $\bigwedge^{2} U$. The formula (1) then shows that $\gamma(U, t)=0$.

"If". Suppose there exists $U$ such that $\gamma(U, t)=0$. We proceed in several steps.

Step 1 . There exists a linear map $h: U \rightarrow \mathbb{C}$ such that

$$
d\left(u_{1} \wedge u_{2}\right)=h\left(u_{2}\right) d_{t}\left(u_{1}\right)-h\left(u_{1}\right) d_{t}\left(u_{2}\right)
$$

for all $u_{1}, u_{2} \in U$.

Indeed, suppose that there exists a nonzero element $\tilde{\gamma} \in \bigwedge^{p+1} W \cong W^{\vee}$ such that $\gamma$ is the image of $\tilde{\gamma}$ under the Koszul differential. Then $\gamma$ coincides with the composition of maps

$$
\bigwedge^{2} W \stackrel{\delta}{\rightarrow} W \otimes W \stackrel{\tilde{\gamma} \otimes \mathrm{id}}{\longrightarrow} W \hookrightarrow V .
$$

Since

$$
\begin{aligned}
d\left(u_{1} \wedge u_{2}\right) & =\gamma\left(d_{t}\left(u_{1}\right) \wedge d_{t}\left(u_{2}\right)\right) \\
& \left.=\tilde{\gamma}\left(d_{t}\left(u_{2}\right)\right) d_{t}\left(u_{1}\right)-\tilde{\gamma}\left(d_{t}\left(u_{1}\right)\right) d_{t}\left(u_{2}\right)\right),
\end{aligned}
$$

condition (5) is satisfied with $h=\tilde{\gamma} \circ d_{t}: U \rightarrow \mathbb{C}$. 
Step 2. Let $u_{1}, u_{2} \in\langle t\rangle \oplus U$ be two sections such that $d_{t}\left(u_{1}\right)$ and $d_{t}\left(u_{2}\right)$ generate $L(-B)$. If $d\left(u_{1} \wedge u_{2}\right)=0$, the extension (4) splits.

To prove this assertion, put $s_{i}=d_{t}\left(u_{i}\right)(i=1,2)$ and consider the commutative diagram

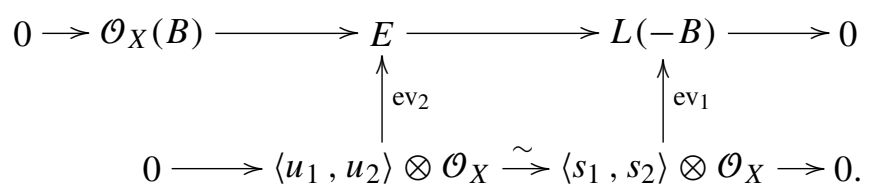

Put $M=\operatorname{ker}\left(\mathrm{ev}_{1}\right)$, and note that $\operatorname{ker}\left(\mathrm{ev}_{2}\right) \cong L^{-1}(B)$ since $\mathrm{ev}_{2}$ is surjective. By the Snake Lemma we obtain an exact sequence

$$
0 \rightarrow M \rightarrow L^{-1}(B) \rightarrow \mathcal{O}_{X}(B) \rightarrow \operatorname{coker}\left(\mathrm{ev}_{1}\right) \rightarrow 0 .
$$

Note that

$$
d\left(u_{1} \wedge u_{2}\right)=0 \Longleftrightarrow \operatorname{rank} \operatorname{im}\left(\left\langle u_{1}, u_{2}\right\rangle \otimes \mathcal{O}_{X} \rightarrow E\right)=1 \Longleftrightarrow \operatorname{rank} M=1
$$

where the first equivalence follows from [10, p. 380]. If $d\left(u_{1} \wedge u_{2}\right)=0$ the above exact sequence shows that $M \cong L^{-1}(B)$, hence the isomorphism $\left\langle u_{1}, u_{2}\right\rangle \otimes \mathcal{O}_{X} \stackrel{\sim}{\rightarrow}$ $\left\langle s_{1}, s_{2}\right\rangle \otimes \mathcal{O}_{X}$ induces an isomorphism $\operatorname{im}\left(\mathrm{ev}_{1}\right) \cong L(-B)$. The inverse of this isomorphism provides a splitting of the extension (4).

Step 3. By Step 1, there exists a linear map $h: U \rightarrow \mathbb{C}$ verifying the relation (5). If $h$ is identically zero, then we can apply Step 1 and Step 2 to conclude. Suppose $h \not \equiv 0$. Consider the morphism

$$
\pi: X \rightarrow \mathbb{P}\left(W^{\vee}\right)
$$

defined by the base-point free linear system $W \subset H^{0}(X, L(-B))$, and choose a linear subspace $\Lambda \subset \mathbb{P}\left(W^{\vee}\right)$ of codimension two such that $\Lambda \cap \pi(X)=\emptyset$. The hyperplane $\operatorname{ker}(h) \subset W$ corresponds to a point $p \in \mathbb{P}\left(W^{\vee}\right)$. Put $H_{1}=\langle\Lambda, p\rangle$ and choose a hyperplane $H_{2} \subset \mathbb{P}\left(W^{\vee}\right)$ containing $\Lambda$ such that $p \notin H_{2}$. Let $u_{1}, u_{2}$ be the sections corresponding to $H_{1}, H_{2}$. Then $d_{t}\left(u_{1}\right)$ and $d_{t}\left(u_{2}\right)$ generate $L(-B)$ and $u_{1} \in \operatorname{ker}(h), u_{2} \notin \operatorname{ker}(h)$. Equation (5) yields the identity

$$
d\left(u_{1} \wedge u_{2}\right)=h\left(u_{2}\right) d_{t}\left(u_{1}\right) .
$$

Rewriting this identity, we obtain $d\left(u_{1} \wedge\left(u_{2}+h\left(u_{2}\right) t\right)\right)=0$. Since the pair $\left\{d_{t}\left(u_{1}\right), d_{t}\left(u_{2}+h\left(u_{2}\right) t\right)\right\}=\left\{d_{t}\left(u_{1}\right), d_{t}\left(u_{2}\right)\right\}$ generates $L(-B)$, Step 2 implies that the extension (4) splits.

Remark 3.2. In the statement of Theorem 3.1 it is not necessary to suppose that $L$ is globally generated, since $K_{p, 1}(X, L(-\mathrm{Bs}(L))) \cong K_{p, 1}(X, L)$. 
Theorem 3.1 yields a short, geometric proof of the Green-Lazarsfeld nonvanishing theorem for curves.

Theorem 3.3 (Green-Lazarsfeld). Let $X$ be a smooth curve, and let $L$ be a line bundle on $X$ that admits a decomposition $L=L_{1} \otimes L_{2}$ with $r_{i}=\operatorname{dim}\left|L_{i}\right| \geq 1$ for $i=1$, 2. Then $K_{r_{1}+r_{2}-1,1}(X, L) \neq 0$.

Proof. We define $s_{1}, s_{2}, t, W, B$ and $\gamma(W, t)$ as in Section 2.2. Let $C$ be the base locus of $W$, seen as a subspace of $H^{0}(X, L(-B))$. We prove that $\gamma(W, t) \neq 0$. Suppose that $\gamma(W, t)=0$. Consider the extension

$$
0 \rightarrow \mathcal{O}_{X}(B) \rightarrow L_{1} \oplus L_{2} \rightarrow L(-B) \rightarrow 0
$$

Pulling back this extension along the injective homomorphism $L(-B-C) \rightarrow$ $L(-B)$, we obtain an induced extension

$$
0 \rightarrow \mathcal{O}_{X}(B) \rightarrow E \rightarrow L(-B-C) \rightarrow 0 .
$$

Applying Theorem 3.1 to the line bundle $L(-C)$, we find that this extension splits. Hence there exists an injective homomorphism

$$
\mathcal{O}_{X}(B) \oplus L(-B-C) \rightarrow L_{1} \oplus L_{2} .
$$

In particular there exists $i \in\{1,2\}$ such that $\operatorname{Hom}\left(L(-B-C), L_{i}\right) \neq 0$. This implies that

$$
r_{i}+1=h^{0}\left(X, L_{i}\right) \geq h^{0}(X, L(-B-C)) \geq \operatorname{dim} W=r_{1}+r_{2}+1,
$$

and this is impossible since $r_{1} \geq 1$ and $r_{2} \geq 1$.

Theorem 3.4. Let $X$ be a smooth curve, and let $\alpha \neq 0 \in K_{p, 1}(X, L)$ be a Koszul class of rank $p+2$ represented by an element of $\bigwedge^{p} W \otimes H^{0}(X, L)$ with $\operatorname{dim} W=p+2$. There exist a rank 2 vector bundle $E$ on $X$, a section $t \in H^{0}(X, E)$ and a subspace $W \cong U \subset H^{0}(X, E)$ such that $\alpha=\gamma(U, t)$.

Proof. Put $T=\mathbb{C} \oplus W$, and choose a basis $\left\{e_{1}, \ldots, e_{p+3}\right\}$ of $T$ such that $t=e_{1}$ is the generator of the first summand. Writing $z_{i j}=e_{i} \wedge e_{j}$, we obtain a skewsymmetric matrix $Z=\left(z_{i j}\right)$ and coordinates $\left(z_{i j}\right)_{1 \leq i<j \leq p+3}$ on $\mathbb{P}\left(\bigwedge^{2} T^{\vee}\right)$. Consider the Grassmannian $G=G(2, T)$ of 2-dimensional quotients of $T$. The ideal of $G$ under the Plücker embedding $G \subset \mathbb{P}\left(\bigwedge^{2} T^{\vee}\right)$ is generated by the $4 \times 4$ Pfaffians $\operatorname{Pf}_{i j k l}(Z)$ of the matrix $Z$. Taking exterior powers in the exact sequence

$$
0 \rightarrow\langle t\rangle \rightarrow T \rightarrow W \rightarrow 0
$$


we obtain an exact sequence

$$
0 \rightarrow\langle t\rangle \otimes W \rightarrow \bigwedge^{2} T \rightarrow \bigwedge^{2} W \rightarrow 0 .
$$

The linear subspace $\mathbb{P}\left(\bigwedge^{2} W^{\vee}\right) \subset \mathbb{P}\left(\bigwedge^{2} T^{\vee}\right)$ is defined by the vanishing of the linear forms $z_{1 j}, j=2, \ldots, p+3$. A straightforward computation then shows that the ideal of the union

$$
G(2, T) \cup \mathbb{P}\left(\bigwedge^{2} W^{\vee}\right) \subset \mathbb{P}\left(\bigwedge^{2} T^{\vee}\right)
$$

is generated by the Pfaffians $\operatorname{Pf}_{1 i j k}(Z)$. The tautological exact sequence

$$
0 \rightarrow S \rightarrow T \otimes \mathcal{O}_{G} \rightarrow Q \rightarrow 0
$$

induces an isomorphism $T \cong H^{0}(G, Q)$. Under this isomorphism, we have $G(2, W)=V(t)$.

As in Section 2.3 we associate to the Koszul class $\alpha$ a matrix $A=\left(a_{i j}\right)$ of linear forms such that

(a) the linear forms in the first row of $A$ span $W$;

(b) there exists a nonzero $4 \times 4$ Pfaffian of $A$ involving the first row and column;

(c) the $4 \times 4$ Pfaffians involving the first row and column of $A$ vanish on the image of $X$ in $\mathbb{P} H^{0}(X, L)^{\vee}$.

Let $C$ be the base locus of the image of $A$. Replacing $L$ by $L(-C$ ) if necessary ( $W$ is obviously contained in the image of $A$ ) we can suppose that $C$ is empty, hence the matrix $A$ defines a morphism

$$
\psi: X \rightarrow \mathbb{P}\left(\bigwedge^{2} T^{\vee}\right)
$$

Condition (c) implies that the image $Y=\psi(X)$ is contained in the union $G(2, T) \cup$ $\mathbb{P}\left(\bigwedge^{2} W^{\vee}\right)$, and condition (a) shows that $Y$ is not contained in $\mathbb{P}\left(\bigwedge^{2} W^{\vee}\right)$. As $Y$ is irreducible, this implies that $Y$ is contained in $G(2, T)$.

Put $E=\psi^{*} Q$. Twisting the exact sequence

$$
0 \rightarrow \mathcal{I}_{Y} \rightarrow \mathcal{O}_{G} \rightarrow \psi_{*} \mathcal{O}_{X} \rightarrow 0
$$

by the universal quotient bundle $Q$ and taking global sections, we obtain an exact sequence

$$
0 \rightarrow H^{0}\left(G, Q \otimes I_{Y}\right) \rightarrow H^{0}(G, Q) \stackrel{\psi^{*}}{\longrightarrow} H^{0}\left(G, \psi_{*} \mathcal{O}_{X} \otimes Q\right) \cong H^{0}(X, E) .
$$

Condition (a) implies that $Y$ is not contained in $G(2, W)=G(2, T) \cap \mathbb{P}\left(\bigwedge^{2} W^{\vee}\right)$, hence $t$ does not vanish identically on $X$ and defines a global section of $E$. The zero locus of this section is given by the equations $a_{12}=\cdots=a_{1, p+3}=0$, hence 
it coincides with the base locus $B$ of the sublinear system of $|L|$ induced by $W$. Consequently the line bundle $E$ is given by an extension

$$
0 \rightarrow \mathcal{O}_{X}(B) \rightarrow E \rightarrow L(-B) \rightarrow 0 .
$$

Consider the commutative diagram

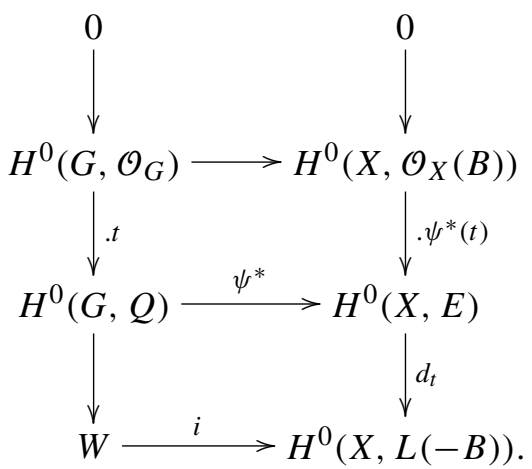

Note that ker $i=W \cap H^{0}\left(G, \mathcal{O}_{G}(1) \otimes \mathcal{I}_{Y}\right)=0$ by condition (a). As the map $H^{0}(G, Q) \rightarrow W$ is surjective, we find that $W$ is contained in the image of the map $d_{t}: H^{0}(X, E) \rightarrow H^{0}(X, L(-B))$. The embedding $W \subset H^{0}(G, Q)=\langle t\rangle \oplus W$ composed with $\psi^{*}$ is a section of $d_{t}$. Put $U=\psi^{*}(W)$. By construction we obtain $\gamma=\gamma(U, t)$.

Remark 3.5. The union $G(2, T) \cup \mathbb{P}\left(\bigwedge^{2} W^{\vee}\right)$ is a generic syzygy scheme; see [6, Theorem 6.1]. In [loc. cit., Theorem 6.7] it was shown that a rank $p+2$ syzygy gives rise to a rank 2 vector bundle if $L$ is very ample and the ideal of $X$ is generated by quadrics.

The condition of Theorem 3.1 can be reinterpreted in terms of surjectivity of a natural multiplication map.

Proposition 3.6. Let $X$ be a smooth curve, and let $W \subset H^{0}(X, L)$ be a linear subspace. We put $B=\mathrm{Bs}(W)$ and view $W$ as a base-point free linear subspace of $H^{0}(X, L(-B))$. Let

$$
\mu: W \otimes H^{0}\left(X, K_{X}(-B)\right) \rightarrow H^{0}\left(K_{X} \otimes L(-2 B)\right)
$$

be the multiplication map. The following conditions are equivalent.

(i) The map $\mu$ is not surjective.

(ii) There exists a non-split extension $0 \rightarrow \mathcal{O}_{X}(B) \rightarrow E \rightarrow L(-B) \rightarrow 0$ such that $W$ is contained in the kernel of the map $\delta: H^{0}(X, L(-B)) \rightarrow H^{1}\left(X, \mathcal{O}_{X}(B)\right)$. 
Proof. We first show that (i) implies (ii). Since $\mu$ is not surjective, there exists a hyperplane $H \subset H^{0}\left(X, K_{X} \otimes L(-2 B)\right)$ that contains $\operatorname{im}(\mu)$. Let $\eta$ be a linear functional defining $H$. Put $0 \neq \xi=\eta^{\vee} \in H^{1}\left(X, L^{-1}(2 B)\right)$, and let

$$
0 \rightarrow \mathcal{O}_{X}(B) \rightarrow E \rightarrow L(-B) \rightarrow 0
$$

be the corresponding non-split extension. Given $w \in W$ and $v \in H^{0}\left(X, K_{X}(-B)\right)$, the formula

$$
\delta(w)(v)=(\eta \circ \mu)(w \otimes v)
$$

shows that $W$ is contained in the kernel of $\delta$.

For the converse, note that formula (7) implies that $\eta \mid \operatorname{im} \mu \equiv 0$.

Remark 3.7. If $B$ is a fixed divisor, the result of the previous Proposition follows from Green's duality theorem [4, Corollary (2.c.10)]. Indeed,

$$
\text { coker } \mu \cong K_{0,1}\left(X, K_{X}(-B), L(-B), W\right) \cong K_{p, 1}(X, B, L(-B), W)^{\vee}
$$

and since $h^{0}\left(X, \mathcal{O}_{X}(B)\right)=1$ we have an injection

$$
K_{p, 1}(X, B, L(-B), W) \hookrightarrow K_{p, 1}(X, L) .
$$

Theorem 3.4 shows that Voisin's method may produce nontrivial Koszul classes that are not contained in the space $K_{p, 1}(X, L)_{\mathrm{GL}}$ spanned by Green-Lazarsfeld classes.

Example 3.8. By [2, Theorem 3.6 and Theorem 4.3] there exists a smooth curve of genus 14 and Clifford index 5 whose Clifford index is computed by a unique line bundle $L$ such that $L^{2}=K_{X}$. The line bundle $L$ embeds $X$ in $\mathbb{P}^{4}$ as a projectively normal curve of degree 13 which is not contained in any quadric of rank $\leq 4$, and the ideal of $X$ is generated by the $4 \times 4$ Pfaffians of a skew-symmetric matrix $\left(a_{i j}\right)_{1 \leq i, j \leq 5}$ with

$$
\operatorname{deg}\left(a_{i j}\right)=\left\{\begin{array}{l}
2 \text { if } i=1 \text { or } j=1 \\
1 \text { if } i \geq 2 \text { and } j \geq 2
\end{array}\right.
$$

such that the quadric $Q=a_{23} a_{45}-a_{24} a_{35}+a_{25} a_{34}$ has rank 5 .

By [loc.cit.] the group $K_{1,1}(X, L)$ is generated by [Q], hence $I_{X}$ contains no quadrics of rank $\leq 4$. If $K_{1,1}(X, L)$ contains a Green-Lazarsfeld class this class would be of scrollar type, since it necessarily comes from two pencils $\left|L_{1}\right|,\left|L_{2}\right|$. This is impossible, since classes of scrollar type give rise to quadrics of rank $\leq 4$.

The Koszul class $[Q] \in K_{1,1}(X, L)$ has rank 3, since it is represented by the linear subspace $W=\left\langle a_{23}, a_{24}, a_{25}\right\rangle$. Hence $[Q]$ comes from Voisin's method by Theorem 3.4. 
Remark 3.9. A more geometric description of a subspace $W$ representing $[Q]$ is the following. A smooth intersection of the quadric $V(Q) \subset \mathbb{P} H^{0}(X, L)^{\vee}$ with one of the cubic Pfaffians is a $K 3$ surface in $\mathbb{P} H^{0}(X, L)^{\vee}$ containing a line $\ell$ which is disjoint from $X$ by [2, Proposition 4.1]. The line $\ell$ corresponds to a 3-dimensional linear subspace $W \subset H^{0}(X, L)$, which is base-point-free since $\ell$ does not meet $X$.

One could ask whether the syzygies constructed in Section 2.1 span $K_{p, 1}(X, L)$. In principle it may be possible to obtain higher rank syzygies as linear combinations of rank $p+2$ syzygies. However, if $K_{p, 1}(X, L)$ is spanned by a single syzygy of rank $\geq p+3$ this is not possible.

Example 3.10 (Eusen-Schreyer). Eusen and Schreyer [3, Theorem 1.7 (a)] have constructed a smooth curve $X \subset \mathbb{P}^{5}$ of genus 7 and Clifford index 3 embedded by the linear system $\left|K_{X}(-x)\right|$ such that $K_{2,1}\left(X, K_{X}(-x)\right) \cong \mathbb{C}$ is spanned by a syzygy $s_{0}$. The explicit expression for $s_{0}$ given on p. 8 of [loc. cit.] shows that $s_{0}$ is a rank 5 syzygy. Hence $s_{0}$ cannot be obtained by the Green-Lazarsfeld construction or the method of Section 2.1.

Acknowledgements. The first named author was partially supported by a Humboldt Research Fellowship, and by the ANCS contract 2-CEx06-11-20/25.07.06. We would like to thank Université Grenoble 1, I.H.E.S., Universität Bayreuth and Université Lille 1 for hospitality during the first stage of this work.

We thank the referees for several comments that helped to improve the presentation of the paper, and for pointing out an error in the previous version of this paper.

\section{References}

[1] D. Eisenbud, Linear sections of determinantal varieties. Amer. J. Math. 110 (1988), 541-575. Zbl 0681.14028 MR 0944327

[2] D. Eisenbud, H. Lange, G. Martens and F.-O. Schreyer, The Clifford dimension of a projective curve. Compositio Math. 72 (2) (1989), 173-204. Zbl 0703.14020 MR 1030141

[3] F. Eusen and F.-O. Schreyer, A remark on a conjecture of Paranjape and Ramanan. Unpublished preprint (1994), avalaible at http://www.math.uni-sb.de/ ag-schreyer/PS/eus.ps

[4] M. Green, Koszul cohomology and the geometry of projective varieties. J. Differential Geom. 19 (1984), 125-171. Zbl 0559.14008 MR 0739785

[5] M. Green, and R. Lazarsfeld, The nonvanishing of certain Koszul cohomology groups. Appendix to [4].

[6] H.-C. Graf von Bothmer, Generic syzygy schemes. J. Pure Appl. Algebra 208 (2007), 867-876. Zbl 05083166 MR 2283431

[7] J. Koh and M. Stillman, Linear syzygies and line bundles on an algebraic curve. J. Algebra 125 (1) (1989), 120-132. Zbl 0708.14002 MR 1012666 
[8] F.-O. Schreyer, Syzygies of canonical curves and special linear series. Math. Ann. 275 (1986), 105-137. Zbl 0578.14002 MR 0849058

[9] C. Voisin, Déformation des syzygies et théorie de Brill-Noether. Proc. London Math. Soc. (3) 67 (3) (1993), 493-515. Zbl 0823.14004 MR 1238043

[10] C. Voisin, Green's generic syzygy conjecture for curves of even genus lying on a $K 3$ surface. J. Eur. Math. Soc. 4 (4) (2002), 363-404. Zbl 1080.14525 MR 1941089

[11] C. Voisin, Green's canonical syzygy conjecture for generic curves of odd genus. Compositio Math. 141 (5) (2005), 1163-1190. Zbl 1083.14038 MR 2157134

Received June 6, 2005

M. Aprodu, Romanian Academy, Institute of Mathematics "Simion Stoilow", P.O.Box 1-764, 014700, Bucharest, Romania

and

Şcoala Normală Superioară-Bucureşti, Calea Griviţei 21, 010702, Bucharest, Romania

E-mail: Marian.Aprodu@imar.ro

J. Nagel, Université Lille 1, Mathématiques - Bât. M2, 59655 Villeneuve d'Ascq Cedex, France

E-mail: nagel@math.univ-lille1.fr 